\title{
Ensayo clínico aleatorizado para evaluar el efecto de un programa de rehabilitación cardiaca supervisado con ejercicio en el consumo de oxígeno, la función y calidad de vida de pacientes con falla cardiaca crónica

\author{
Luz Helena Lugo ${ }^{a}$, Claudia María Navas ${ }^{\mathrm{b}}$, Jesús A. Plata ${ }^{\mathrm{c}}$, Sergio D. Ortiz ${ }^{\mathrm{b}}$, \\ Domingo Caraballo $^{\mathrm{d}}$, Ana Cecilia Henao ${ }^{e}$, Héctor Iván García ${ }^{\mathrm{f}}$ y Juan M. Sénior ${ }^{\mathrm{g}, \mathrm{e}, \mathrm{h}, *}$
}

a Grupo de Rehabilitación en Salud, Universidad de Antioquia, Medellín, Colombia

${ }^{\mathrm{b}}$ Medicina Física y Rehabilitación, Clínica Las Américas, Medellín, Colombia

c Departamento de Medicina Física y Rehabilitación, Universidad de Antioquia, Medellín, Colombia

d IPS Universitaria, Universidad de Antioquia, Medellín, Colombia

e Hospital Universitario San Vicente Fundación, Medellín, Colombia

f Grupo Académico de Epidemiología Clínica (GRAEPIC) Universidad de Antioquia, Medellín, Colombia

s Posgrado Cardiología Clínica y Cardiología Intervencionista, Universidad de Antioquia, Medellín, Colombia

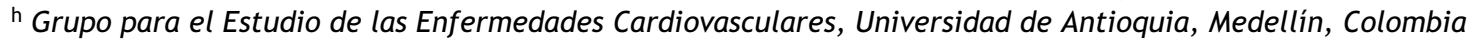

Recibido el 23 de diciembre de 2016; aceptado el 14 de mayo de 2017

Disponible en Internet el 7 de noviembre de 2017

\section{PALABRAS CLAVE}

Falla cardiaca;

Rehabilitación;

Ejercicio;

Calidad de vida

\begin{abstract}
Resumen
Objetivo: evaluar el efecto de un programa supervisado sobre el consumo de oxígeno, la función y la calidad de vida en pacientes con falla cardiaca.

Diseño y métodos: ensayo clínico con doble enmascaramiento de grupos paralelos, en pacientes con falla cardiaca estado funcional II-Iv por más de 6 meses con fracción de eyección $<40 \%$. El desenlace primario fue el consumo de oxígeno pico a las 8 semanas.

Resultados: veintitrés pacientes fueron asignados al grupo de intervención y 26 al grupo de control. Cinco fallecieron, 4 se negaron a completar todas las evaluaciones y 1 no realizó la prueba de ejercicio cardiopulmonar. Diecisiete fueron analizados en el grupo de intervención y 20 en el grupo de control. Respecto $\mathrm{al} \mathrm{VO}_{2}$ pico, no se observaron cambios estadísticamente significativos al final del programa de intervención a las 8 semanas $(-13,3 \pm 3,9 \mathrm{ml} / \mathrm{kg} / \mathrm{min}$ en el grupo de intervención frente a $14,8 \pm 4,6 \mathrm{ml} / \mathrm{kg} / \mathrm{min}$ en el grupo de control, $\mathrm{p}=0,31)$. Las evaluaciones de funcionalidad y calidad de vida no difirieron entre los grupos a las 8 semanas o 6 meses. Sin embargo, hubo una mejoría en ambos grupos en los resultados de funcionalidad y calidad de vida.
\end{abstract}

* Autor para correspondencia.

Correo electrónico: mmbt@une.net.co (J.M. Sénior). 
Conclusión: el uso de un programa de ejercicio protocolizado y supervisado en pacientes con falla cardiaca con una fracción de eyección < 40\%, no produce cambios significativos en el $\mathrm{VO}_{2}$ pico en comparación con un programa comunitario.

(c) 2017 Sociedad Colombiana de Cardiología y Cirugía Cardiovascular. Publicado por Elsevier España, S.L.U. Este es un artículo Open Access bajo la licencia CC BY-NC-ND (http:// creativecommons.org/licenses/by-nc-nd/4.0/).

\section{KEYWORDS}

Heart failure; rehabilitation; exercise; quality of life

\section{A randomised clinical trial to evaluate the effect of a supervised rehabilitation program with exercise on oxygen consumption, function, and quality of life of patients with chronic heart failure}

\begin{abstract}
Objective: To evaluate the effect of a supervised exercise program on oxygen consumption, function, and quality of life in patients with heart failure.

Design and methods: A double-blind clinical trial, with parallel groups, conducted on patients with functional stage II-Iv heart failure for more than 6 months and with an ejection fraction of $<40 \%$. The primary outcome was the peak oxygen consumption at 8 weeks.

Results: A total of 23 patients were assigned to the intervention group and 26 to the control group. Of those, 5 died, 4 failed to complete all the evaluations, and 1 did not perform the cardiopulmonary exercise test. Finally, 17 patients were analysed in the intervention group and 20 in the control group. As regards the peak $\mathrm{VO}_{2}$, no statistically significant changes were observed at the end of the intervention program at 8 weeks $(-13.3 \pm 3.9 \mathrm{ml} / \mathrm{kg} / \mathrm{min}$ in the intervention group compared to $14.8 \pm 4.6 \mathrm{ml} / \mathrm{kg} / \mathrm{min}$ in the control group, $P=.31$ ). There were no differences between the groups in the functional evaluations and the quality of life at 8 weeks or 6 months. However, the results showed an improvement in the functionality and quality of life in both groups.

Conclusion: The use of a standard and supervised exercise program by patients with heart failure with an ejection fraction $<40 \%$ does not lead to significant changes in the peak $\mathrm{VO}_{2}$, when compared to a community program.

(c) 2017 Sociedad Colombiana de Cardiología y Cirugía Cardiovascular. Published by Elsevier España, S.L.U. This is an open access article under the CC BY-NC-ND license (http:// creativecommons.org/licenses/by-nc-nd/4.0/).
\end{abstract}

\section{Introducción}

La falla cardiaca crónica es una condición común y progresiva, caracterizada por síntomas típicos como tolerancia reducida al ejercicio, que se evidencia por fatiga y disnea desencadenadas con un mínimo grado de intensidad de actividad, acompañado por signos como edema periférico, aumento de la presión venosa yugular y crépitos ${ }^{1}$, situación que conduce a un estilo de vida sedentario, deterioro de la independencia funcional ${ }^{2} \mathrm{y}$ de la calidad de vida de quienes la padecen ${ }^{3}$.

Los programas de rehabilitación cardiaca hacen parte del tratamiento integral del paciente con falla cardiaca ${ }^{4}$. Hasta hace aproximadamente diez años, estos pacientes evitaban la práctica de ejercicio por temor a una descompensación; sin embargo, recientemente se han entendido mejor los efectos benéficos del mismo ${ }^{4}$. En el ensayo clínico HF ACTION se demostró que en el grupo de pacientes asignado a entrenamiento con ejercicio hubo un modesto incremento del consumo de oxígeno pico $\left(\mathrm{VO}_{2} \text { pico }\right)^{5}$; además, se relacionó con disminución del desenlace compuesto de muerte de cualquier causa y hospitalización de cualquier causa así como del desenlace secundario de muerte cardiovascular y hospitalización de causa cardiovascular ${ }^{6}$. En otros estudios se demostró que el ejercicio aeróbico no sólo mejora el $\mathrm{VO}_{2}$ pico, sino la fuerza y la masa muscular ${ }^{7}$, la clase funcional medida con la New York Heart Association (NYHA), la calidad de vida y, en algunos casos, la remodelación del ventrículo izquierdo, independiente del tipo de actividad utilizada, aunque algunos sugieren mejor efecto al incluir resistencia ${ }^{8}$.

La depresión es otra comorbilidad importante en los pacientes con falla cardiaca, con una prevalencia cercana al $21,5 \%{ }^{\circ}$. Estos pacientes tienen tendencia a mayor mortalidad, mayor uso de recursos en salud y mayor tasa de hospitalizaciones ${ }^{10}$. El entrenamiento con ejercicio disminuye significativamente los síntomas depresivos en estos pacientes ${ }^{11}$, a diferencia de algunos medicamentos de uso común que no han demostrado ningún efecto ${ }^{12,13}$.

Los programas controlados de ejercicio son seguros; en una revisión de la literatura para evaluar la evidencia de los programas de ejercicios en rehabilitación cardiaca se encontró que la seguridad del ejercicio está bien establecida ${ }^{14}$. Según una revisión sistemática por la Colaboración Cochrane con 2.172 participantes donde comparan programas de rehabilitación cardiaca dirigidos institucionalmente con programas realizados en casa, se demuestra que no hay diferencias en cuanto a la mortalidad y eventos cardiacos o 
capacidad de ejercicio ${ }^{15}$; estos datos fueron corroborados por otro meta-análisis con diseño similar ${ }^{16}$.

El propósito de este estudio fue establecer el efecto en el consumo de oxígeno, la funcionalidad y la calidad de vida en un grupo de pacientes con falla cardiaca crónica a quienes se les realizó un programa de ejercicio protocolizado y supervisado, comparado con un programa de ejercicio basado en la comunidad con las recomendaciones usuales. El estudio se diseñó como ensayo clínico con asignación aleatoria, de superioridad, con grupos paralelos, enmascarado para el evaluador de los desenlaces y evaluación del desenlace primario a las 8 semanas.

El presente reporte es el análisis pre-especificado del estudio Natriuretic Brain Pro-peptid Changes in Patients With Chronic Heart Failure Within a Cardiac Rehabilitation Program con registro en clinicaltrials.gov (número NCT02087670).

\section{Pacientes y métodos}

\section{Población}

Se incluyeron pacientes mayores de 18 años con diagnóstico de falla cardiaca de más de seis meses de evolución que acudieron a una Clínica de Falla Cardiaca de una institución de alta complejidad de la ciudad de Medellín, Antioquia, que no tuvieran criterios de exclusión y aceptaran participar. Se excluyeron pacientes que estuvieran en clase funcional NYHA I, que hubiesen asistido a un programa de rehabilitación cardiaca, diabetes mellitus descompensada, hipertensión arterial de difícil control, enfermedad cardiaca isquémica en el último mes, embolia pulmonar previa, enfermedad pulmonar obstructiva crónica, enfermedad restrictiva pulmonar, estenosis valvular aórtica, episodio nuevo de fibrilación auricular y enfermedades osteomusculares que limitaran la actividad física.

Este estudio fue aprobado por el comité de ética del Centro de Investigaciones Médicas de la Universidad de Antioquia, teniendo en cuenta la declaración de Helsinki y similares, emanados de la Asociación Médica Mundial, y se ajusta a la resolución 8430 de 1993 del Ministerio de Salud de la República de Colombia, clasificándose como de riesgo mínimo; se veló por la confidencialidad de la información, la veracidad en los resultados obtenidos de ésta y el respeto por los derechos de los pacientes objeto de estudio, los cuales ingresaron luego de firmar el consentimiento informado.

\section{Diseño}

Ensayo clínico, prospectivo, con asignación al azar y enmascarado para los profesionales que evaluaron los desenlaces y para quien hizo el análisis estadístico. Los pacientes elegidos fueron asignados por medio de una secuencia de números aleatorios generados por un computador y además se utilizó el sistema de sobres cerrados y opacos. Los pacientes se asignaron a uno de los siguientes dos grupos: grupo intervención (grupo 1) y grupo de control (grupo 2). El tamaño de muestra para un estudio de medidas repetidas tuvo en cuenta un error $\alpha$ 0,05, error $\beta$ 0,2, 1 medición antes de hacer la asignación y dos mediciones después; una diferencia clínica estandarizada por el estadístico de Cohen de 0,61, y un índice de correlación entre las mediciones de 0,6 . Con estos parámetros se tuvo un tamaño de muestra de 38 pacientes más un $20 \%$ de pérdidas por posible falta de adherencia al protocolo, comorbilidades asociadas, complicaciones propias de la enfermedad y hospitalizaciones, lo que arrojó un total de 46 pacientes; sin embargo, fue posible incluir 49 pacientes.

La capacidad de ejercicio aeróbico, la calidad de vida, la funcionalidad y la depresión fueron evaluadas al inicio, luego del programa de intervención o a las ocho semanas y a los seis meses. A los pacientes del grupo control se les permitió realizar la actividad física que hacían normalmente, además se les educó en el control de los factores de riesgo: control lipídico, manejo de la hipertensión (medicación), cambio en el hábito del consumo de cigarrillo, control del peso, manejo de la diabetes y control psicológico; adicionalmente, se les brindó orientación y apoyo ocupacional. Los pacientes del grupo de intervención, además de las actividades educativas recibieron un programa de rehabilitación cardiaca supervisado por ocho semanas en una Unidad de Rehabilitación Cardiaca de un centro de alta complejidad que dispone de clínica de falla cardiaca. Todos los pacientes continuaron la medicación y los cuidados de la dieta recomendada.

El programa de intervención consistió en sesiones de ejercicio aeróbico cardiovascular dos veces por semana, con una mediana de asistencia a 14 sesiones durante las ocho semanas, monitorizadas con telemetría, control de la presión arterial y de la frecuencia cardiaca, las cuales fueron supervisadas por un médico especialista en medicina física y rehabilitación y una enfermera entrenados en rehabilitación cardiaca. Cada sesión tuvo una duración aproximada de una hora, inicio con cinco minutos de calentamiento, seguido de entrenamiento en una banda sinfín, a una intensidad de ejercicio que oscilaba entre el 60 y el $80 \%$ de la capacidad de ejercicio aeróbico del paciente, la cual se determinó durante la primera sesión del programa, con la aplicación de un protocolo de Bruce modificado o a una intensidad de ejercicio entre 12 y 16 en la escala de percepción del ejercicio de Borg; la sesión finalizaba con cinco minutos de enfriamiento.

Los pacientes recibieron además, una sesión de terapia física por semana, durante el mismo tiempo, en las cuales se realizó un entrenamiento segmentario de la fuerza muscular, con una intensidad del $60 \%$ de una repetición máxima. Se hizo énfasis en el fortalecimiento de los grupos musculares funcionales y de los anti-gravitatorios, y se tuvo en cuenta la no realización de maniobra de Valsalva durante el ejercicio; también recibieron un promedio de dos sesiones de terapia ocupacional, en las cuales se brindó educación sobre técnicas de ahorro energético y recomendaciones ergonómicas.

\section{Evaluaciones y desenlaces del estudio}

A todos los pacientes se les hizo una evaluación inicial, a las ocho semanas y a los seis meses, que incluía una historia clínica completa y una clasificación funcional de acuerdo con la NYHA.

El desenlace principal fue el consumo de oxigeno pico $\left(\mathrm{VO}_{2}\right)$, que se evaluó antes de iniciar el programa, a las ocho semanas o luego de terminar el programa de intervención 


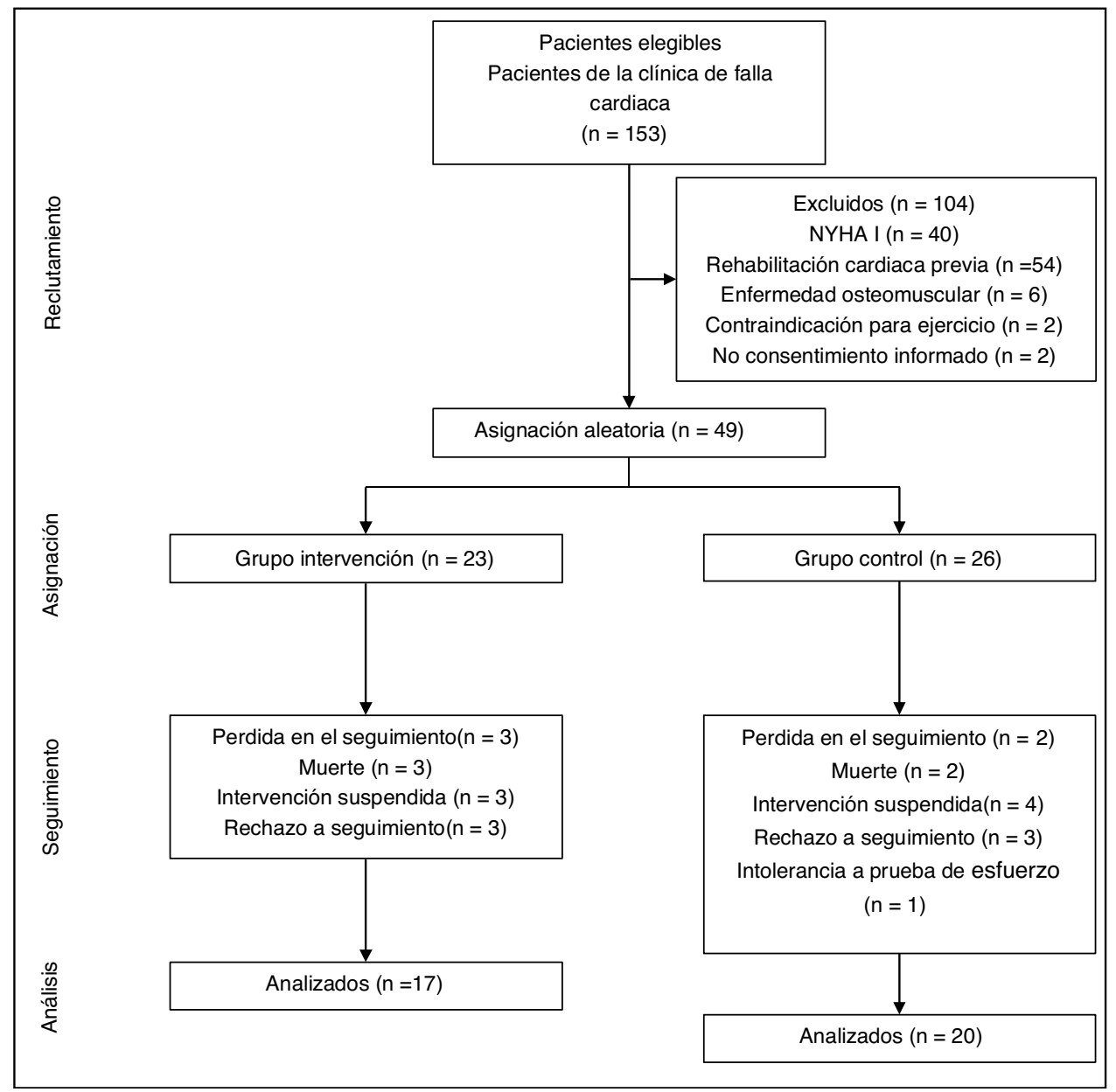

Figura 1 Diagrama de flujo. NYHA: clasificación de falla cardiaca New York Heart Association.

y a los seis meses. $\mathrm{El} \mathrm{VO}_{2}$ se determinó con una prueba de esfuerzo cardiopulmonar, la cual estuvo a cargo de un médico fisiólogo, especialista en pruebas de esfuerzo, en un ergo-espirómetro marca Quinton Metabolic Cart Q710, utilizando un protocolo de aumento de la resistencia de 17 Vatios cada tres minutos; el $\mathrm{VO}_{2}$ pico fue determinado con el promedio de las mediciones de los últimos 30 segundos del ejercicio, mientras que el umbral anaerObico (UA) fue determinado de forma no invasiva por el método v-Slope. Las medidas determinadas en el pico del ejercicio y el umbral anaerObico fueron la ventilación pulmonar (VE), el $\mathrm{VO}_{2}$ y la producción de dióxido de carbono $\left(\mathrm{VCO}_{2}\right)$.

Los desenlaces secundarios medidos fueron los metros recorridos durante una prueba de marcha de seis minutos (6MWT), el puntaje del SF-36 y de la prueba de Zung y la medición de algunas variables ecocardiográficas. La prueba de 6 MWT, una medida de la capacidad funcional, se hizo al inicio, a las ocho semanas o luego de terminar el programa de intervención y a los seis meses, y fue llevada a cabo por un médico entrenado en su aplicación, quien determinó la distancia recorrida.
La evaluación de la calidad de vida se hizo basalmente, a las ocho semanas o luego de terminar el programa de intervención y a los seis meses, y se utilizó la escala SF36, la cual fue validada para Colombia. Ésta mide diez dimensiones: función física (FF), rol físico (RF), dolor corporal (DC), salud general (SG), vitalidad (VT), función social (FS), rol emocional (RE), salud mental (SM), y un ítem adicional de cambio en la percepción del estado de salud actual; se evalúa de 0-100 (el máximo valor indica estar mejor). En forma simultánea con el SF-36 se aplicó la escala de Zung, la cual puede categorizar en depresión leve, moderada y grave o ausencia de síntomas.

En la evaluación basal y a los seis meses, se realizó ecocardiografía con características Modo $\mathrm{M}$, bidimensional, doppler color, las cuales estuvieron a cargo de un médico cardiólogo, especialista en ecocardiografía, en un equipo Hewlett-Packard Sonos 5.500, en el laboratorio de ecocardiografía que cumple con las normas de la Sociedad Americana de Ecocardiografía y del American College of Cardiology (ACC). Las variables que se determinaron fueron el diámetro sistólico del ventrículo izquierdo, el diámetro 
Tabla 1 Características epidemiológicas de los pacientes

\begin{tabular}{llcrr}
\hline Características & & Grupo intervención & Grupo control & Total \\
\hline Sexo & Masculino & $12(52,2 \%)$ & $14(53,8 \%)$ & $26(53,1 \%)$ \\
& Femenino & $11(47,8 \%)$ & $12(46,2 \%)$ & $23(46,9 \%)$ \\
Clase funcional & 2 & $2(9,1 \%)$ & $5(20,8 \%)$ & $7(15,2 \%)$ \\
& 3 & $15(68,2 \%)$ & $14(58,3 \%)$ & $29(63,0 \%)$ \\
& 4 & $5(22,7 \%)$ & $5(20,8 \%)$ & $10(21,7 \%)$
\end{tabular}

Características

Grupo intervención

Grupo control

\begin{tabular}{|c|c|c|c|c|}
\hline & & & \\
\hline & $\mathrm{n}$ & Media (desviación estándar) & $n$ & Media (desviación estándar) \\
\hline Edad & 23 & $54,4(13,6)$ & 26 & $56,5(11,0)$ \\
\hline IMC & 23 & $22,7(3,6)$ & 25 & $23,8(3,1)$ \\
\hline \multicolumn{5}{|l|}{ Ecocardiografía } \\
\hline Diámetro sistólico del vı & 21 & $5,8(0,9)$ & 23 & $5,4(1,2)$ \\
\hline Volumen sistólico del vı & 21 & $178,2(64,1)$ & 22 & $147,9(68,8)$ \\
\hline Volumen diastólico del vı & 21 & $230,6(85,7)$ & 22 & $204,5(104,8)$ \\
\hline Diámetro diastólico del vi* & 21 & $6,6(2,2-8,7)$ & 23 & $6,4(2,4-8,3)$ \\
\hline Fracción de eyección" & 21 & $30,0(20,0-60,0)$ & 23 & $35,0(25,0-75,0)$ \\
\hline Diámetro del VD" & 21 & $1,7(1,2-4,4)$ & 23 & $2,0(0,8-2,4)$ \\
\hline Fracción de acortamiento* & 21 & $15,0(8,0-30,0)$ & 23 & $18,0(13,0-30,0)$ \\
\hline \multicolumn{5}{|l|}{ Prueba de esfuerzo } \\
\hline $\mathrm{VO}_{2}$ pico $(\mathrm{ml} / \mathrm{kg} / \mathrm{min})$ & 22 & $13,3(3,1)$ & 24 & $12,7(3,6)$ \\
\hline $\mathrm{VO}_{2}$ pico $(\mathrm{ml} / \mathrm{min})$ & 22 & $814,2(224,7)$ & 24 & $804,8(222,1)$ \\
\hline $\mathrm{UA}\left(\% \mathrm{VO}_{2} \text { pico }\right)^{*}$ & 20 & $71,5(24,0-98,0)$ & 21 & $75,0(57,0-94,0)$ \\
\hline MET" & 22 & $3,8(0,9)$ & 24 & $3,7(1,0)$ \\
\hline \multicolumn{5}{|c|}{ Test de Zung y caminata de 6 minutos } \\
\hline Test Zung* & 23 & $37,5(28,7-77,5)$ & 26 & $39,4(31,2-56,2)$ \\
\hline Distancia en metros* & 22 & $354(140-445)$ & 26 & $333(120-501)$ \\
\hline \multicolumn{5}{|l|}{ SF-36 Calidad de vida } \\
\hline Dolor corporal ${ }^{*}$ & 23 & $100(22-100)$ & 26 & $79(41-100)$ \\
\hline Cambio de salud & 23 & $60(20-100)$ & 26 & $80(20-100)$ \\
\hline Desempeño emocional ${ }^{*}$ & 23 & $66(0-100)$ & 26 & $100(0-100)$ \\
\hline Desempeño físico* & 23 & $50(0-100)$ & 26 & $25(0-100)$ \\
\hline Función física* & 23 & $70(30-100)$ & 26 & $70(15-95)$ \\
\hline Función social" & 23 & $100(25-200)$ & 26 & $100(37-100)$ \\
\hline Salud mental" & 23 & $72(8-100)$ & 26 & $78(28-100)$ \\
\hline Vitalidad" & 23 & $65(5-100)$ & 26 & $72,5(25-100)$ \\
\hline Salud general ${ }^{*}$ & 23 & $61,1(25,6)$ & 26 & $63,1(22,5)$ \\
\hline
\end{tabular}

* Mediana (mínimo-máximo)

diastólico del ventrículo izquierdo, la fracción de eyección y la fracción de acortamiento.

\section{Análisis estadístico}

Las variables cualitativas se describieron por medio de frecuencias y las variables cuantitativas por medio de promedios con sus desviaciones estándar o con las medianas y los valores máximos y mínimos, de acuerdo con la distribución. Inicialmente se analizaron las variables cuantitativas y se estableció su normalidad por medio de la prueba de Kolmogorov-Smirnov. Se elaboró un análisis por medio de una comparación de medianas con la prueba de Wilcoxon; para el análisis de las variables categóricas se utilizó una comparación de frecuencias por medio de una prueba de $\mathrm{Chi}^{2}$.

Para evaluar la variabilidad de los grupos en los diferentes momentos (inicial, 2 meses y 6 meses), se utilizó un modelo de ecuaciones estimables generalizadas para el análisis de medidas repetidas, pues las medidas no son independientes entre sí y no necesariamente tienen distribución normal. El tamaño del efecto se evaluó por medio del estadístico de Cohen, el cual determina la magnitud de la diferencia de los promedios entre los grupos (antes y después del tratamiento) teniendo en cuenta la desviación estándar de cada uno y el número de pacientes por grupo; los califica como un efecto mínimo $(\geq-0,15 \mathrm{y}<.15)$, pequeño $(\geq .15 \mathrm{y}<.40)$, medio $(\geq .40 \mathrm{y}<.75)$, grande $(\geq .75 \mathrm{y}<1,10)$, muy grande $(\geq 1,10$ y $<1,45)$ y gigante $(>1,45)$. Se realizó un análisis por tratamiento. 


\section{Resultados}

Luego de la asignación aleatoria 23 pacientes ingresaron al grupo de intervención y 26 al grupo control (fig. 1). Durante el seguimiento, cinco pacientes fallecieron; cuatro rehusaron completar todas las evaluaciones y uno no logró realizar la prueba de esfuerzo cardiopulmonar, la mitad de los pacientes del grupo de intervención asistieron a 140 más sesiones de ejercicio; el resto asistieron a 13 sesiones o menos. No hubo diferencias significativas en las características demográficas, clínicas ni ecocardiográficas entre ambos grupos al inicio (tabla 1). No se presentaron complicaciones durante los programas de entrenamiento ni efectos adversos durante el seguimiento. El $84,78 \%$ de los pacientes se clasificaba en las clases funcionales III $(63,04 \%)$ y iv $(21,74 \%)$. La fracción de eyección en promedio fue de $34,9 \%$; para la clase funcional III de $33,14 \%$ y para la iv de $29,6 \%$.

El consumo de oxígeno y los MET fueron evaluados como parámetros de ejercicio. (tabla 2), en los que no se encontraron cambios estadísticamente significativos al terminar el programa de intervención a los dos meses ni en el seguimiento a los seis meses. La distancia recorrida durante la prueba de marcha de los seis minutos (6 MWT) y el puntaje total de la escala de Zung no presentaron diferencias.

La calidad de vida en el grupo de intervención tuvo variaciones estadísticamente significativas en las dimensiones de cambio en la percepción de salud, RE, RF, FF, SM y SG al comparar la evaluación a los dos meses y la evaluación inicial. Los aspectos más importantes al evaluar la magnitud del cambio fueron, en orden de importancia, la función física, el rol físico, el cambio de salud y el rol emocional. Aunque la vitalidad mejoró no fue un cambio significativo; el dolor corporal tampoco cambió pero se resalta que inicialmente esta dimensión estaba en el nivel máximo. En el grupo control no hubo cambios en ninguna de las dimensiones del SF-36 (tabla 3).

De las variables ecocardiográficas analizadas sólo el diámetro sistólico, el volumen sistólico del ventrículo izquierdo y la fracción de eyección tuvieron un cambio significativo en el grupo control, al comparar la evaluación a los seis meses con la evaluación inicial (tabla 4).

Al evaluar el tamaño del efecto con el estadístico de cohen (tabla 5), en el análisis de los modelos de ecuaciones estimables generalizadas en las tres mediciones durante todo el seguimiento, se encontraron diferencias clínicas mínimas (al tener en cuenta el tamaño del efecto) en el test de Zung, la prueba de marcha de los seis minutos y en el SF36 para función social, vitalidad y desempeño emocional, y diferencias clínicas pequeñas entre los grupos en los demás desenlaces, pues el valor del estadístico es menor de 0,4. Los estimadores que se muestran en la tabla 5 hacen referencia al promedio de las mediciones entre los pacientes y entre los grupos durante los seis meses de seguimiento. En el caso de la prueba de los seis minutos (6MWT), por ejemplo, se puede decir que los pacientes del grupo de intervención caminaron en promedio 8,11 metros más que los pacientes del grupo control en el promedio a los seis meses de seguimiento; sin embargo este valor no es estadísticamente significativo. El desenlace del consumo de oxígeno $(\mathrm{ml} / \mathrm{min})$ en el grupo de intervención, disminuyó $56,25 \mathrm{ml} / \mathrm{min}$ en promedio al compararlo con los pacientes del grupo de cuidado

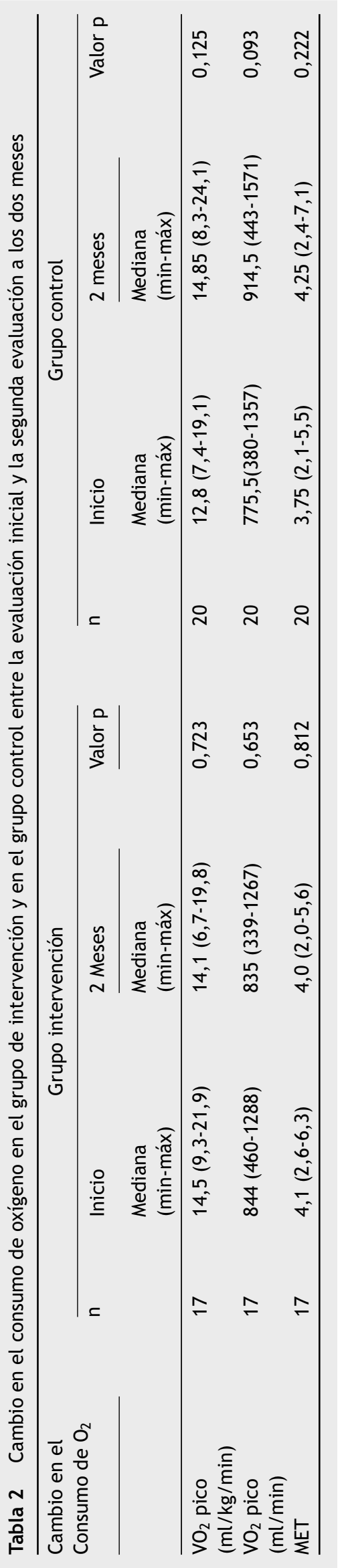


Tabla 3 Comparación de los resultados del test de Zung y las escalas de calidad de vida del S-F36 entre la evaluación inicial y los dos meses en ambos grupos

\begin{tabular}{|c|c|c|c|c|c|c|c|c|}
\hline \multirow{3}{*}{$\begin{array}{l}\text { Comparación test de } \\
\text { Zung S-F36 }\end{array}$} & \multicolumn{4}{|c|}{ Grupo intervención } & \multicolumn{4}{|c|}{ Grupo control } \\
\hline & \multirow[t]{2}{*}{$n$} & \multirow{2}{*}{$\begin{array}{l}\text { Inicial } \\
\text { Mediana } \\
\text { (Mínimo-Máximo) }\end{array}$} & \multirow{2}{*}{$\begin{array}{l}2 \text { meses } \\
\text { Mediana } \\
\text { (Mínimo-Máximo) }\end{array}$} & \multirow[t]{2}{*}{ Valor $\mathrm{p}$} & \multirow[t]{2}{*}{$\mathrm{N}$} & \multirow{2}{*}{$\begin{array}{l}\text { Inicial } \\
\text { Mediana } \\
\text { (Mínimo-Máximo) }\end{array}$} & \multirow{2}{*}{$\begin{array}{l}2 \text { meses } \\
\text { Mediana } \\
\text { (Mínimo-Máximo) }\end{array}$} & \multirow[t]{2}{*}{ Valor $\mathrm{p}$} \\
\hline & & & & & & & & \\
\hline Test zung & 17 & $37,5(28,7-77,5)$ & $35,6(28,7-53,7)$ & 0,069 & 22 & $39,4(31,2-56,2)$ & $35,6(28,7-53,7)$ & 0.174 \\
\hline $\begin{array}{l}\text { Distancia en metros } \\
\text { S-F36 }\end{array}$ & 16 & $360(140-445)$ & $372,5(300-522)$ & 0,120 & 21 & $333(120-501)$ & $360(120-510)$ & 0,348 \\
\hline Dolor corporal & 17 & $100(52-100)$ & $100(22-100)$ & 1,0 & 22 & $79(41-100)$ & $100(22-100)$ & 0,576 \\
\hline Cambio de salud & 17 & $60(20-100)$ & $100(20-100)$ & 0,007 & 22 & $80(20-100)$ & $100(20-100)$ & 0,431 \\
\hline Desempeño emocional & 17 & $66(33-100)$ & $100(33-100)$ & 0,011 & 22 & $100(0-100)$ & $100(0-100)$ & 0,450 \\
\hline Desempeño físico & 17 & $50(0-100)$ & $100(0-100)$ & 0,006 & 22 & $25(0-100)$ & $50(0-100)$ & 0,205 \\
\hline Función física & 17 & $80(30-100)$ & $85(65-100)$ & 0,024 & 22 & $70(35-95)$ & $77,5(10-100)$ & 0,175 \\
\hline Función social & 17 & $100(25-100)$ & $100(100-100)$ & 0,109 & 22 & $100(37-100)$ & $100(50-100)$ & 0,857 \\
\hline Salud mental & 17 & $72(24-100)$ & $88(48-100)$ & 0,009 & 22 & $78(32-100)$ & $84(32-100)$ & 0,981 \\
\hline Vitalidad & 17 & $65(30-100)$ & $75(45-100)$ & 0,657 & 22 & $72.5(30-100)$ & $75,5(20-100)$ & 0,855 \\
\hline Salud general & 17 & $70(5-95)$ & $80(5-100)$ & 0,01 & 22 & $62,5(25-95)$ & $65(0-100)$ & 0,410 \\
\hline
\end{tabular}

Mediana (mínimo - máximo), prueba de Wilcoxon

Tabla 4 Comparación de las variables ecocardiográficas entre la evaluación inicial y los seis meses, en ambos grupos

\begin{tabular}{|c|c|c|c|c|c|c|c|c|}
\hline \multirow{3}{*}{$\begin{array}{l}\text { Comparación de } \\
\text { las variables } \\
\text { ecocardiográficas }\end{array}$} & \multicolumn{4}{|c|}{ Grupo intervención } & \multicolumn{4}{|c|}{ Grupo control } \\
\hline & \multirow[t]{2}{*}{$n$} & \multirow{2}{*}{$\begin{array}{l}\text { Inicial } \\
\text { Mediana } \\
\text { (mínimo-máximo) }\end{array}$} & \multirow{2}{*}{$\begin{array}{l}6 \text { meses } \\
\text { Mediana } \\
\text { (mínimo-máximo) }\end{array}$} & \multirow[t]{2}{*}{ Valor $\mathrm{p}$} & \multirow[t]{2}{*}{$\mathrm{n}$} & \multirow{2}{*}{$\begin{array}{l}\text { Inicial } \\
\text { Mediana } \\
\text { (mínimo-máximo) }\end{array}$} & \multirow{2}{*}{$\begin{array}{l}6 \text { meses } \\
\text { Mediana } \\
\text { (mínimo-máximo) }\end{array}$} & \multirow[t]{2}{*}{ Valor $\mathrm{p}$} \\
\hline & & & & & & & & \\
\hline $\begin{array}{l}\text { Diámetro sistólico } \\
\text { del vı }\end{array}$ & 17 & $5,6(4,9-7,7)$ & $6,0(4,1-7,8)$ & 0,254 & 16 & $5,6(3,4-8,2)$ & $4,4(3,2-7,7)$ & 0,036 \\
\hline $\begin{array}{l}\text { Diámetro } \\
\text { diastólico del vı }\end{array}$ & 17 & $2,0(1,4-3,2)$ & $1,6(1,0-2,6)$ & 0,462 & 16 & $6,6(2,4-8,3)$ & $5,6(5,0-8,8)$ & 0,339 \\
\hline $\begin{array}{l}\text { Fracción de } \\
\text { eyección }\end{array}$ & 18 & $30,0(20-40)$ & $33(15-55)$ & 0,329 & 16 & $30(25-50)$ & $40(26-60)$ & 0,020 \\
\hline Diámetro del VD & 15 & $2,0(1,4-3,2)$ & $1,6(1-2,6)$ & 0,231 & 16 & $2,1(1,0-2,4)$ & $1,7(1,0-2,3)$ & 0,422 \\
\hline $\begin{array}{l}\text { Fracción de } \\
\text { acortamiento }\end{array}$ & 18 & $13(10-15)$ & $18,5(10-20)$ & 0,119 & 16 & $15(13-25)$ & $15(13-25)$ & 0,133 \\
\hline
\end{tabular}

Tabla 5 Modelos de ecuaciones estimables generalizables para evaluación de los desenlaces a seis meses

\begin{tabular}{lcl}
\hline Prueba de esfuerzo & Estimador (IC 95\%) & Cohen \\
\hline $\mathrm{VO}_{2}$ pico $(\mathrm{ml} / \mathrm{kg} / \mathrm{min})$ & $-0,82(-2,84-1,2)$ & 0,2 \\
$\mathrm{VO}_{2}$ pico $(\mathrm{ml} / \mathrm{min})$ & $-56,25(-199,67-86,67)$ & 0,2 \\
$\mathrm{MET}$ & $-0,256(-0,854-0,34)$ & 0,21 \\
Test zung & $-0,57(-5,14-4,01)$ & 0,06 \\
Distancia en metros (6 MWT) & $8,11(-34,12-50,32)$ & 0,1 \\
SF-36 Calidad de vida & & \\
Dolor corporal & $8,05(-3,77-19,87)$ & 0,31 \\
Cambio de salud & $-7,98(-18,87-2,92)$ & 0,31 \\
Desempeño emocional & $3,56(-11,22-18,34)$ & 0,11 \\
Desempeño físico & $8,68(-8,31-25,66)$ & 0,23 \\
Función física & $4,65(-4,54-13,84)$ & 0,24 \\
Función social & $1,89(-6,46-10,26)$ & 0,1 \\
Salud mental & $-4,62(-14,94-5,69)$ & 0,22 \\
Vitalidad & $-3,02(-13,94-7,89)$ & 0,14 \\
Salud general & $4,41(-7,48-16,3)$ & 0,19 \\
\hline
\end{tabular}

usual en el promedio de los seis meses de seguimiento, pero este hallazgo no fue estadísticamente significativo.

\section{Discusión}

Los resultados indican que luego de un programa de intervención de ejercicio protocolizado y supervisado de dos meses de duración no se presentaron diferencias clínicas ni estadísticamente significativas en el consumo de oxígeno pico al compararlo con un programa basado en la comunidad con las recomendaciones usuales.

Estudios previos han demostrado que los programas de rehabilitación cardiaca producen cambios centrales y periféricos, los cuales mejoran la capacidad funcional y la percepción del estado de salud, al permitir mayores cargas de ejercicio con menor elevación de la frecuencia cardiaca $^{17}$, aunque estos efectos pueden perderse si el entrenamiento no se mantiene a largo plazo ${ }^{18}$. El ejercicio mejora el $\mathrm{VO}_{2}$ pico entre $18-25 \%$ y la duración del pico de ejercicio entre 18 a $34 \%{ }^{17}$, sin embargo el incremento 
parece estar directamente relacionado con la intensidad del ejercicio; Ismail et al. demostraron en una revisión sistemática que después de entrenamiento de alta intensidad (> $85 \% \mathrm{VO}_{2}$ pico) se produce incremento del $\mathrm{VO}_{2}$ pico de $3,9 \mathrm{ml} / \mathrm{kg} /$ minuto en comparación con ejercicio vigoroso (60-85\% $\mathrm{VO}_{2}$ pico), de moderada intensidad $\left(40-59 \% \mathrm{VO}_{2}\right.$ pico) y de baja intensidad ( $<40 \% \mathrm{VO}_{2}$ pico), que produce elevaciones discretas de $2,8 \mathrm{ml} / \mathrm{kg} /$ minuto, $2,2 \mathrm{ml} / \mathrm{kg} /$ minuto y $1,0 \mathrm{ml} / \mathrm{kg} /$ minuto, respectivamente ${ }^{19}$. Estos hallazgos fueron corroborados por metarregresión en el estudio de Uddin et al., en la que se demostró que solo la intensidad del ejercicio se asocia de manera significativa con el $\mathrm{VO}_{2}$ máximo ${ }^{20}$. Aunque la intensidad definida en este estudio se considera dentro del rango vigoroso, no se demostraron cambios significativos en el $\mathrm{VO}_{2}$ pico a los dos y seis meses de seguimiento en ninguno de los dos grupos, ni a los seis meses al compararlos, pese a que en el grupo control hubo incremento de éste y del número de MET alcanzados.

Los pacientes con falla cardiaca avanzada tienen un $\mathrm{VO}_{2}$ pico que puede diferenciar la pobre capacidad funcional y aunque no existe un punto de corte óptimo, un nivel menor de $18,3 \mathrm{ml} / \mathrm{kg} /$ minuto parece distinguir dos grupos diferentes; este hecho explicaría por qué muchas actividades de la vida diaria implican un esfuerzo cercano al máximo, con gran costo de consumo de oxígeno, lo que lleva a desacondicionamiento severo ${ }^{21}$. La mayoría de los pacientes del estudio se encontraba en clase funcional avanzada y aunque está claro que se relaciona con el $\mathrm{VO}_{2}$ pico $^{22}$, es poco probable que incida en la respuesta al ejercicio, para explicar completamente los resultados obtenidos, máxime cuando se instauró un programa integral de rehabilitación, por lo que cobra relevancia la incompetencia cronotrópica y los factores periféricos ${ }^{23}$.

La adherencia al tratamiento es una variable a tener en cuenta, incluyendo las recomendaciones de ejercicio ${ }^{24}$; la mitad de los pacientes logró cumplir con más de 14 sesiones de entrenamiento y la otra mitad 13 sesiones o menos a pesar de los esfuerzos realizados por el ensayo, lo que se convierte en un factor importante al evaluar los resultados. El estudio HF ACTION demostró relación del volumen de ejercicio tanto con el $\mathrm{VO}_{2}$ pico como con desenlaces clínicamente importantes, aunque niveles bajos también demostraron efecto benéfico ${ }^{25}$; Belardinelli et al. demostraron en un seguimiento a 10 años, que el ejercicio moderado supervisado mantiene la capacidad funcional y la calidad de vida, con reducciones en los eventos cardiovasculares mayores ${ }^{26}$. Por lo anterior, está claro que la no adherencia a las medidas no farmacológicas, incluyendo el ejercicio, se relaciona con desenlaces adversos ${ }^{27}$. Son muchas las barreras descritas para la implementación de un programa de ejercicio supervisado; entre las más importantes están el nivel educativo y el género, además de algunos aspectos motivacionales, económicos y socioculturales ${ }^{28}$, que podrían impactarse con programas estructurados específicamente para mejorar la adherencia de los pacientes y concientizar a las familias y al sistema de salud acerca de la importancia de estos ${ }^{29}$.

Al evaluar la calidad de vida relacionada con la salud, por medio de una escala genérica como lo es el SF-36, se encontró un cambio estadísticamente significativo en la mayoría de dimensiones de la escala a favor del grupo de intervención a los dos meses, el cual se ha reportado en otros estudios previos ${ }^{30}$, especialmente con alta intensidad de ejercicio ${ }^{31}$. Los resultados del estudio indican que un programa de entrenamiento aeróbico permite mejorar la percepción de la calidad de vida relacionada con la salud a corto plazo, aunque al evaluarlo en la totalidad del seguimiento no hay cambios significativos entre los grupos.

Es importante tener en cuenta que la que se usó en este estudio es una escala general de calidad de vida, la cual tiene propiedades sicométricas adecuadas ${ }^{32}$ y posee la ventaja teórica que permite establecer comparaciones de los desenlaces en un espectro de diferentes enfermedades; sin embargo, su capacidad para medir la sensibilidad al cambio es moderada en comparación con escalas específicas ${ }^{33}$; con alta correlación en la validez de constructo y confiabilidad con escalas como la de Minnesota Living with Heart Failure Questionnaire (MLHFQ) y Kansas City Cardiomyopathy Questionnaire (KCCQ), que son las más recomendadas ${ }^{34}$. A pesar de lo anterior y al extenso uso de estas escalas, ninguna de ellas satisface los requisitos de la Food and Drug Administration (FDA) ${ }^{35}$.

La depresión es una comorbilidad frecuente en pacientes con falla cardiaca y las estrategias para manejarla son poco efectivas ${ }^{36}$. En el presente estudio no se demostró reducción significativa en el puntaje total de la escala de depresión de Zung en ninguno de los dos grupos; un metaanálisis de ensayos clínicos demostró reducción en los síntomas de depresión en pacientes con falla cardiaca consecutiva, a través del análisis de múltiples subgrupos, incluyendo programas supervisados en centros o en casa ${ }^{11}$.

El proceso de remodelación ventricular es uno de los factores claves en la progresión de los pacientes con falla cardiaca y se relaciona con factores hemodinámicos, neuroendocrinos y energéticos ${ }^{37}$. Se logró demostrar una tendencia hacia el incremento de la fracción de eyección en ambos grupos, con significancia estadística en el grupo control, al igual que en el diámetro sistólico del ventrículo izquierdo, solamente en este último grupo. Estos resultados pudiesen estar explicados porque el tamaño de muestra no se calculó específicamente para corroborar una mejoría en la remodelación ventricular y porque los estudios que han demostrado mejoría en estos aspectos han requerido programas de intervención de mayor duración (> 6 meses $)^{38}$.

Una fortaleza importante del presente reporte es la inclusión, en estudios longitudinales de estas características, del análisis por medio de modelos de ecuaciones estimables generalizadas para medidas repetidas, ya que estas se enfocan en datos correlacionados de varios individuos en un mismo grupo, más que en las variaciones de las observaciones longitudinales del mismo individuo, con lo que se obtienen estimativos más eficientes de los parámetros de regresión, menos sesgados y coeficientes de regresión con errores estándar exactos, que permiten obtener intervalos de confianza correctos ${ }^{39}$.

\section{Limitaciones}

La principal limitación del estudio está en relación con la adherencia de los pacientes a las medidas no farmacológicas, en este caso particular al entrenamiento con ejercicio, reportado consistentemente en la literatura y de origen multifactorial; de igual forma, no seguir las recomendaciones 
de las guías de práctica clínica a largo plazo tendría efectos deletéreos, los cuales no fueron controlados durante el ensayo. Otra consideración radica en que el programa de ejercicio supervisado se realizó en banda sinfín y la prueba de esfuerzo cardiopulmonar en cicloergómetro, lo que pudiese cambiar las condiciones de carga y por lo tanto la percepción de la fatiga, logrando menores niveles de ejercicio, a pesar de cumplir con los lineamientos establecidos para estas pruebas.

\section{Conclusión}

No hay diferencias en el consumo de oxígeno ni en la funcionalidad con la implementación de un programa de corta duración de ejercicio protocolizado y supervisado al compararlo con un programa de ejercicio basado en la comunidad con las recomendaciones usuales en pacientes con falla cardiaca. El programa mejoró la calidad de vida en los componentes físicos y mentales a las ocho semanas cuando terminó el entrenamiento, pero no se mantuvo a los seis meses.

\section{Responsabilidades éticas}

Protección de personas y animales. Los autores declaran que los procedimientos seguidos se conformaron a las normas éticas del comité de experimentación humana responsable y de acuerdo con la Asociación Médica Mundial y la Declaración de Helsinki.

Confidencialidad de los datos. Los autores declaran que han seguido los protocolos de su centro de trabajo sobre la publicación de datos de pacientes.

Derecho a la privacidad y consentimiento informado. Los autores han obtenido el consentimiento informado de los pacientes y/o sujetos referidos en el artículo. Este documento obra en poder del autor de correspondencia.

\section{Financiación}

Comité para el Desarrollo de la Investigación, Universidad de Antioquia (CODI), proyectos de mediana cuantía.

\section{Conflictos de interes y aspectos éticos}

Ensayo clínico aleatorizado, controlado para evaluar el efecto de un programa de rehabilitación cardiaca supervisado con ejercicio en el consumo de oxígeno, la función y calidad de vida de pacientes con falla cardiaca crónica.

La investigación fue financiada con recursos por convocatoria del Comité para el Desarrollo de la Investigación Universidad de Antioquia -CODI-, proyectos de mediana cuantía y no hay conflictos de interés por declarar.

Los aspectos éticos se ajustan a los principios éticos fundamentales y a las directrices de la Declaración de Helsinki y similares, emanados de la Asociación Médica Mundial, y sigue las pautas del Ministerio de la Protección Social de la República de Colombia según la resolución 8430 de 1993 por la cual se dictan las normas científicas, técnicas y administrativas para la investigación en salud.

\section{Bibliografía}

1. Ponikowski P, Voors AA, Anker SD, Bueno H, Cleland JG, Coats AJ, et al. 2016 ESC Guidelines for the diagnosis and treatment of acute and chronic heart failure: The Task Force for the diagnosis and treatment of acute and chronic heart failure of the European Society of Cardiology (ESC). Developed with the special contribution of the Heart Failure Association (HFA) of the ESC. Eur J Heart Fail. 2016;18:891-975.

2. Pollack T, Madigan E. Functional status outcome measures in home health care patients with heart failure. Home Health Care Serv Q. 2010;29:155-70.

3. Nieminen MS, Dickstein K, Fonseca C, Serrano JM, Parissis J, Fedele F, et al. The patient perspective: Quality of life in advanced heart failure with frequent hospitalisations. Int J Cardiol. 2015;191:256-64.

4. Taylor RS, Sagar VA, Davies EJ, Briscoe S, Coats AJ, Dalal H, et al. Exercise-based rehabilitation for heart failure. Cochrane Database Syst Rev. 2014. CD003331.

5. O'Connor CM, Whellan DJ, Lee KL, Keteyian SJ, Cooper LS, Ellis SJ, et al. HF-ACTION Investigators. Efficacy and safety of exercise training in patients with chronic heart failure: HF-ACTION randomized controlled trial. JAMA. 2009;301:1439-50.

6. Swank AM, Horton J, Fleg JL, Fonarow GC, Keteyian S, Goldberg $\mathrm{L}$, et al. Modest increase in peak VO2 is related to better clinical outcomes in chronic heart failure patients: results from heart failure and a controlled trial to investigate outcomes of exercise training. Circ Heart Fail. 2012;5:579-85.

7. De Maeyer C, Beckers P, Vrints C, Conraads V. Exercise training in chronic heart failure. Ther Adv Chronic Dis. 2013;4:105-17.

8. Haykowsky MJ, Liang Y, Pechter D, Jones LW, McAlister FA, Clark AM. A meta-analysis of the effect of exercise training on left ventricular remodeling in heart failure patients: the benefit depends on the type of training performed. J Am Coll Cardiol. 2007; 49:2329-36.

9. Rutledge T, Reis VA, Linke SE, Greenberg BH, Mills PJ. Depression in heart failure a meta-analytic review of prevalence, intervention effects, and associations with clinical Outcomes. J Am Coll Cardiol. 2006;48:1527-37.

10. Sherwood A, Blumenthal JA, Hinderliter AL, Koch GG, Adams KF Jr, Dupree CS, et al. Worsening depressive symptoms are associated with adverse clinical outcomes in patients with heart failure. J Am Coll Cardiol. 2011;57:418-23.

11. Tu RH1, Zeng ZY, Zhong GQ, Wu WF, Lu YJ, Bo ZD, et al. Effects of exercise training on depression in patients with heart failure: a systematic review and meta-analysis of randomized controlled trials. Eur J Heart Fail. 2014;16:749-57.

12. Angermann CE, Gelbrich G, Störk S, Gunold H, Edelmann F, Wachter R, et al. Effect of escitalopram on all-cause mortality and hospitalization in patients with heart failure and depression: The MOOD-HF Randomized Clinical Trial. JAMA. 2016;315:2683-93.

13. O.Connor CM, Jiang W, Kuchibhatla M, Silva SG, Cuffe MS, Callwood DD, et al. Safety and efficacy of sertraline for depression in patients with heart failure: results of the SADHART-CHF (Sertraline Against Depression and Heart Disease in Chronic Heart Failure) trial. J Am Coll Cardiol. 2010;56:692-9.

14. Sagar VA, Davies EJ, Briscoe S, Coats AJ, Dalal HM, Lough F, et al. Exercise-based rehabilitation for heart failure: systematic review and meta-analysis. Open Heart. 2015;2:e000163.

15. Taylor RS, Dalal H, Jolly K, Zawada A, Dean SG, Cowie A, Norton RJ. Home-based versus centre-based cardiac rehabilitation. Cochrane Database Syst Rev. 2015. CD007130. 
16. Buckingham SA, Taylor RS, Jolly K, Zawada A, Dean SG, Cowie A, et al. Home-based versus centre-based cardiac rehabilitation: abridged Cochrane systematic review and meta-analysis. Open Heart. 2016;3:e000463.

17. Downing J, Balady GJ. The role of exercise training in heart failure. J Am Coll Cardiol. 2011;58:561-9.

18. Belardinelli R, Georgiou D, Cianci G, Purcaro A. Randomized, controlled trial of long-term moderate exercise training in chronic heart failure: effects on functional capacity, quality of life, and clinical outcome. Circulation. 1999;99:1173-82.

19. Ismail H, McFarlane JR, Nojoumian AH, Dieberg G, Smart NA. Clinical outcomes and cardiovascular responses to different exercise training intensities in patients with heart failure: a systematic review and meta-analysis. JACC Heart Fail. 2013;1:514-22.

20. Uddin J, Zwisler AD, Lewinter C, Moniruzzaman M, Lund K, Tang LH, Taylor RS. Predictors of exercise capacity following exercise-based rehabilitation in patients with coronary heart disease and heart failure: A meta-regression analysis. Eur J Prev Cardiol. 2016;23:683-93.

21. Morey MC, Pieper CF, Cornoni-Huntley J. Is there a threshold between peak oxygen uptake and self-reported physical functioning in older adults? Med Sci Sports Exerc. 1998;30:1223-9.

22. Russell SD, Saval MA, Robbins JL, Ellestad MH, Gottlieb SS, Handberg EM, et al. New York Heart Association functional class predicts exercise parameters in the current era. Am Heart J. 2009;158 4 Suppl:S24-30.

23. Shimiaie J, Sherez J, Aviram G, Megidish R, Viskin S, Halkin $A$, et al. Determinants of Effort Intolerance in Patients With Heart Failure: Combined Echocardiography and Cardiopulmonary Stress Protocol. JACC Heart Fail. 2015;3:803-14.

24. Deka P, Pozehl B, Williams MA, Yates B. Adherence to recommended exercise guidelines in patients with heart Failure. Heart Fail Rev. 2016 Sep 26 [Epub ahead of print] DOI: 10.1007/s10741016-9584-1.

25. Keteyian SJ, Leifer ES, Houston-Miller N, Kraus WE, Brawner CA, O'Connor CM, et al. Relation between volume of exercise and clinical outcomes in patients with heart failure. J Am Coll Cardiol. 2012;60:1899-905.

26. Belardinelli R, Georgiou D, Cianci G, Purcaro A. 10-year exercise training in chronic heart failure: a randomized controlled trial. J Am Coll Cardiol. 2012;60:1521-8.

27. van der Wal MH, van Veldhuisen DJ, Veeger NJ, Rutten FH, Jaarsma T. Compliance with non-pharmacological recommendations and outcome in heart failure patients. Eur Heart J. 2010;31:1486-93.
28. Klompstra L, Jaarsma T, Stromberg A. Physical activity in patients with heart failure: barriers and motivations with special focus on sex differences. Patient Prefer Adherence. 2015;9:1603-10.

29. Pozehl BJ, Duncan K, Hertzog M, McGuire R, Norman JF, Artinian NT, et al. Study of adherence to exercise in heart failure: the HEART camp trial protocol. BMC Cardiovasc Disord. 2014;14:172.

30. Flynn KE, Piña IL, Whellan DJ, Lin L, Blumenthal JA, Ellis SJ, et al. Effects of exercise training on health status in patients with chronic heart failure: HF-ACTION randomized controlled trial. JAMA. 2009;301:1451-9.

31. Chrysohoou C, Tsitsinakis G, Vogiatzis I, Cherouveim E, Antoniou C, Tsiantilas A, et al. High intensity, interval exercise improves quality of life of patients with chronic heart failure: a randomized controlled trial. QJM. 2014;107:25-32.

32. McHorney CA, Ware JE, Raczek AE. The MOS 36-item short-form health survey (SF-36). II. Psychometric and clinical tests of validity in measuring physical and mental health constructs. Med Care. 1993;31:247-63.

33. Dunderdale K, Thompson DR, Miles JN, Beer SF, Furze G. Quality-of-life measurement in chronic heart failure: do we take account of the patient perspective? Eur J Heart Fail. 2005; 7:572-82.

34. Garin O, Ferrer M, Pont A, Rué M, Kotzeva A, Wiklund I, et al. Disease-specific health-related quality of life questionnaires for heart failure: a systematic review with meta-analyses. Qual Life Res. 2009;18:71-85.

35. Psotka MA, von Maltzahn R, Anatchkova M, Agodoa I, Chau D, Malik FI, et al. Patient-Reported Outcomes in Chronic Heart Failure: Applicability for Regulatory Approval. JACC Heart Fail. 2016;4:791-804.

36. Ghosh RK, Ball S, Prasad V, Gupta A. Depression in heart failure: Intricate relationship, pathophysiology and most updated evidence of interventions from recent clinical studies. Int $J$ Cardiol. 2016;224:170-7.

37. Bernardo BC, McMullen JR. Molecular aspects of exerciseinduced cardiac remodeling. Cardiol Clin. 2016;34:515-30.

38. Chen YM, Li ZB, Zhu M, Cao YM. Effects of exercise training on left ventricular remodelling in heart failure patients: an updated meta-analysis of randomised controlled trials. Int J Clin Pract. 2012;66:782-91.

39. Hanley JA, Negassa A, Edwardes MD, Forrester JE. Statistical analysis of correlated data using generalized estimating equations: an orientation. Am J Epidemiol. 2003;157:364-75. 